\title{
Liraglutide improves cognitive impairment via the AMPK and PI3K/Akt signaling pathways in type 2 diabetic rats
}

\author{
YING YANG ${ }^{1}$, HUI FANG $^{1,2}$, GANG XU $^{3}$, YANFENG ZHEN ${ }^{2}$, YAZHONG ZHANG ${ }^{2}$, \\ JINLI TIAN $^{2}$, DANDAN ZHANG ${ }^{2}$, GUYUE ZHANG $^{2}$ and JING XU ${ }^{2}$ \\ ${ }^{1}$ Department of Internal Medicine, Hebei Medical University, Shijiazhuang, Hebei 050017; ${ }^{2}$ Second Department of \\ Endocrinology; ${ }^{3}$ Department of Burns and Orthopedics, Tangshan Gongren Hospital, Tangshan, Hebei 063000, P.R. China
}

Received October 13, 2017; Accepted February 14, 2018

DOI: $10.3892 / \mathrm{mmr} .2018 .9180$

\begin{abstract}
Liraglutide is a type of glucagon-like-peptide 1 receptor agonist, which has been reported as a novel type of antidiabetic agent with numerous benefits, including cardiovascular and neuroprotective effects. To the best of our knowledge, few studies to date have reported the potential mechanism underlying the neuroprotective effects of liraglutide on rats with type 2 diabetes mellitus (T2DM). The present study aimed to investigate the neuroprotective actions of liraglutide in diabetic rats and to determine the mechanisms underlying these effects. A total of 30 male T2DM Goto-Kakizaki (GK) rats (age, 32 weeks; weight, 300-350 g) and 10 male Wistar rats (age, 32 weeks; weight, 300-350 g) were used in the present study. Wistar rats received vehicle treatment, and GK rats randomly received treatment with vehicle, low dose of liraglutide $(75 \mu \mathrm{g} / \mathrm{kg})$ or high dose of liraglutide $(200 \mu \mathrm{g} / \mathrm{kg})$ for 28 days. Cognitive deficits were evaluated using the Morris water maze test. The expression levels of phosphoinositide 3-kinase (PI3K), protein kinase B (Akt), phosphorylated (p)-Akt, AMP-activated protein kinase (AMPK), mammalian target of rapamycin (mTOR), Beclin-1, microtubule-associated protein light chain 3 (LC)-3 II, caspase-3, B-cell lymphoma 2 (Bcl-2)-associated X protein and $\mathrm{Bcl}-2$ were assessed by western blot analysis. The results demonstrated that diabetic GK rats exhibited cognitive dysfunction, whereas treatment with liraglutide alleviated the learning and memory deficits, particularly in the high-dose liraglutide group. The expression levels of Beclin-1 and LC-3 II were decreased in GK rats; however, this decrease was alleviated in the presence of liraglutide. Liraglutide also reversed T2DM model-induced increases in mTOR, and decreases in p-AMPK, PI3K and p-Akt expression, and modulated the
\end{abstract}

Correspondence to: Professor Hui Fang, Department of Internal Medicine, Hebei Medical University, 361 Zhongshan East Road, Shijiazhuang, Hebei 050017, P.R. China

E-mail: fanghui2818@126.com

Key words: diabetes, liraglutide, autophagy, apoptosis, cognitive deficits expression of apoptosis-associated proteins. Furthermore, the administration of liraglutide inhibited apoptosis and exerted a protective effect against cognitive deficits via the activation of autophagy. In conclusion, the protective effects of liraglutide may be associated with increased mTOR expression via activation of the AMPK and PI3K/Akt signaling pathways.

\section{Introduction}

Type 2 diabetes mellitus (T2DM) is a widespread chronic metabolic disorder, which can adversely affect numerous tissues due to its long-term complications. In addition to common complications in the peripheral nervous system of patients with T2DM (1), emerging evidence had indicated that T2DM exerts negative effects on the central nervous system (CNS), with cognitive impairment the most common symptom (2). 'Diabetes-associated cognitive decline (DACD)' has been proposed as a novel term to strengthen research in associated fields and to facilitate recognition of this disorder (3). Based on clinical, epidemiological and experimental evidence, numerous studies have suggested a causal association between T2DM and cognitive impairment (4-6). Therefore, DM-induced cognitive impairment has received increasing attention; however, the pathophysiological alterations and pathogenesis remain to be investigated. Cognitive dysfunction in DM appears to be caused by various factors (7). Recent studies demonstrated that T2DM could result in autophagic dysfunction in neuronal cells, thus aggravating cerebral vascular disease and the process of vascular dementia, eventually resulting in memory dysfunction $(8,9)$. Autophagy serves an important role in maintaining normal tissue and cellular homeostasis (10). A previous study revealed the crucial role of autophagy in DM, in which autophagy functions to ameliorate or exacerbate DM-induced cognitive dysfunction $(9,11)$. Therefore, regulation of autophagy in the hippocampus may be considered an essential method to ameliorate neuronal damage.

Considering the pathogenesis of T2DM and brain dysfunction, antidiabetic drugs may also exert beneficial effects on neuronal metabolism, which could be clinically significant for the treatment of CNS complications in DM and other neurological diseases (12). Glucagon-like peptide-1 (GLP-1) receptor agonists are a novel type of hypoglycemic drug used in clinical practice, which have been reported to possess numerous 
potential benefits in improving capacity in various animal models of neurodegeneration (13-15). In the Guangxi Diabetes and Metabolic Disorders (GDMD) Study in China, clinical studies demonstrated that plasma dipeptidyl peptidase 4 (DPP4) activity, which is associated with the degradation of GLP-1, had a positive association with mild cognitive impairment in elderly patients with T2DM (16). In addition, it has been revealed that DPP4 inhibitors may improve cognitive deficits through inhibiting oxidative stress or the inflammatory reaction in diverse mouse models $(17,18)$. Among the GLP-1 receptor agonists, liraglutide is a long-acting GLP-1 receptor agonist, which has a 97\% amino acid sequence similarity with human GLP-1 and exhibits an increased half-life of $13 \mathrm{~h}$ (19). Liraglutide has been reported to prevent memory impairments in object recognition and water maze tasks. Simultaneously it prevents synaptic loss and impairment of synaptic plasticity in the hippocampus of mice with Alzheimer's disease (AD) (20). Furthermore, a previous study suggested that liraglutide acts to increase neuronal density and improve cognitive function in the hippocampus of a senescence-accelerated mouse model of AD (21). Another study confirmed that liraglutide can activate autophagic pathways in diabetic rats (22). However, the possibility of administrating liraglutide for improving cognitive function and the potential underlying mechanism warrants further investigation. In the present study, GK rats were used to investigate the effect of numerous doses of liraglutide on cognitive improvement, and to gain deeper insight into the molecular mechanisms underlying autophagy. In combination with other evidence (23), the present study hypothesized that liraglutide is a candidate cognitive enhancer that acts by increasing mammalian target of rapamycin (mTOR) expression via the AMP-activated protein kinase (AMPK) and phosphoinositide 3 kinase (PI3K)/protein kinase B (Akt) pathways. The present study may provide novel evidence to suggest liraglutide exerts therapeutic effects on T2DM-induced cognitive deficits.

\section{Materials and methods}

Animals. The animal experiments were conducted in compliance with the guidelines for the Care and Use of Laboratory Animals (24). The present study was approved by the experimental ethics committee of the North China University of Science and Technology (Tangshan, China). A total of 30 male Goto-Kakizaki (GK) rats (age, 32 weeks; weight, 300-350 g) and 10 male Wistar rats (age, 32 weeks; weight, 300-350 g) were obtained from the Animal Center of North China University of Science and Technology. The rats were maintained in plastic cages $(\mathrm{n}=5$ rats/cage) with a temperature range of $20-24^{\circ} \mathrm{C}$ and humidity of $50 \pm 10 \%$, under a 12 -h light/dark cycle. The rats were permitted free access to food and water.

Preparation of animal models. A total of $30 \mathrm{GK}$ rats were randomly divided into three groups ( $\mathrm{n}=10$ rats/group): DM group, DM + low dose of liraglutide group [DM + Lira-L, $75 \mu \mathrm{g} / \mathrm{kg}$ subcutaneous (sc.)] and DM + high dose of liraglutide group (DM + Lira-H, $200 \mu \mathrm{g} / \mathrm{kg}$ sc.). Liraglutide was administered once a day between days 1 and 28. The liraglutide dosing was gradually increased from 37.5 to $75 \mu \mathrm{g} / \mathrm{kg}$ in the $\mathrm{DM}+$ Lira-L group $(37.5,75,75$ and $75 \mu \mathrm{g} / \mathrm{kg}$ respectively), and from 37.5 to $200 \mu \mathrm{g} / \mathrm{kg}$ in the DM + Lira-H group (37.5, 75,150 and $200 \mu \mathrm{g} / \mathrm{kg}$ respectively) over the first 4 days. GK rats in the DM group and 10 Wistar rats in the control group were administered $0.9 \%$ sterile saline ( $75 \mu \mathrm{g} / \mathrm{kg} \mathrm{sc}$.). Following treatment, behavioral tests and biochemical experiments were performed.

Morris water maze (MWM) test. The spatial learning and memory of all rats were tested in the MWM 28 days following liraglutide and saline treatments. Rats were placed in a black circular pool filled with water (diameter, $180 \mathrm{~cm}$; depth, $45 \mathrm{~cm}$ ), which was virtually divided into four equivalent quadrants: North, south, east and west; the water temperature was maintained at $24-26^{\circ} \mathrm{C}$. The reference objects around the water tank were considered to be visual hints and remained unaltered throughout the MWM test. During the process of testing, the rats were randomly placed into the water in any of the four quadrants facing the wall of the pool. The test was conducted for 4 days, with one test performed in each quadrant every morning for $60 \mathrm{sec}$. The rats that could not find the platform or failed to escape within $90 \mathrm{sec}$ were placed on the platform and allowed to stand for $15 \mathrm{sec}$, and the measurement was recorded as $90 \mathrm{sec}$. Those that found the platform were also permitted to stand on it for $15 \mathrm{sec}$. On day 5, the platform was removed and the rats were allowed to swim for $1 \mathrm{~min}$. Maze performance was recorded using a video camera located above the tank, which was interfaced with a video tracking system (HVS Image Software Ltd., Hampton, UK). The average escape latency of a total of five trials and the time rats remained in the target quadrant were calculated.

Histology. Following the behavioral tests, all rats were anesthetized with sodium pentobarbital $(60 \mathrm{mg} / \mathrm{kg})$ and euthanized by transcardiac perfusion with cold PBS and then perfused with cold $4 \%$ paraformaldehyde containing $0.2 \%$ saturated picric acid in PBS. The hippocampus was removed and collected from the two hemispheres. Some samples were frozen at $-80^{\circ} \mathrm{C}$. The remaining samples were post-fixed overnight at $4^{\circ} \mathrm{C}$ in the same fixative solution. The post-fixed samples were embedded in paraffin and cut along the sagittal plane at a thickness of $5 \mu \mathrm{m}$ using a microtome. Paraffin-embedded brain sections were treated with xylene to remove paraffin and were rehydrated, after which they were stained with $0.1 \%$ $(\mathrm{w} / \mathrm{v})$ cresyl violet for $10 \mathrm{~min}$ at $37^{\circ} \mathrm{C}$. The severity of neuronal damage was evaluated by counting the number of surviving neurons under an optical microscope (Olympus Corporation, Tokyo, Japan). The mean number of morphologically intact neurons was calculated and recorded per $100 \mu \mathrm{m}$ in the CA1 hippocampal area, in order to accurately estimate the degree of neuronal damage.

Western blot analysis. Frozen hippocampal samples were obtained and lysed in Tissue Protein Lysis Solution (Thermo Fisher Scientific, Inc., Waltham, MA, USA) which contained 5\% Proteinase Inhibitor Cocktail (Sigma-Aldrich; Merck KGaA, Darmstadt, Germany). Protein concentration was determined by the BCA reagent method (Wuhan Boster Biological Technology Ltd., Wuhan, China). Equivalent quantities of protein $(30 \mu \mathrm{g})$ were separated by $12 \%$ SDS-PAGE (Beyotime Institute of Biotechnology, Haimen, China) and 
underwent western blot analysis. Following electrophoresis, the proteins were transferred onto polyvinylidene difluoride membranes (Beyotime Institute of Biotechnology) using a wet transfer system, the membranes were then blocked in 5\% fat-free dry milk for $2 \mathrm{~h}$ at room temperature and washed three times in PBS with $0.1 \%$ Tween 20 at room temperature. The membrane was incubated overnight at $4^{\circ} \mathrm{C}$ with the following primary antibodies: Rabbit polyclonal anti-PI3K p110 antibody (1:3,000; cat. no. 4255; Cell Signaling Technology, Inc., Danvers, MA, USA), rabbit polyclonal anti-p-Akt antibody (1:1,000; cat. no. ab38449; Abcam, Cambridge, UK), rabbit polyclonal anti-Akt antibody $(1: 1,000$; cat. no. ab8805; Abcam), rabbit polyclonal anti-p-AMPK antibody $(1: 1,000$; cat. no. ab23875; Abcam), rabbit polyclonal anti-AMPK antibody (1:1,000; cat. no. ab131512; Abcam), rabbit polyclonal anti-p-mTOR antibody (1:1,000; cat. no. ab84400; Abcam), rabbit polyclonal anti-mTOR antibody (1:1,000; cat. no. ab2732; Abcam), rabbit polyclonal anti-caspase-3 antibody (1:1,000; cat. no. ab13847; Abcam), rabbit monoclonal anti-B-cell lymphoma 2 (Bcl-2)-associated X protein (Bax) antibody (1:1,000; cat. no. ab32503; Abcam), rabbit polyclonal anti-Bcl-2 antibody (1:1,000; cat. no ab59348; Abcam), rabbit polyclonal anti-Beclin-1 antibody (1:1,000; cat. no. ab62557; Abcam), rabbit polyclonal anti-microtubule-associated protein 1 light chain 3 I (LC-3 I) antibody (1:1,000; cat. no. ab62720; Abcam), rabbit polyclonal anti-microtubule-associated protein 1 light chain 3 II (LC-3 II) antibody (1:1,000; cat. no. ab51520; Abcam) and rabbit anti- $\beta$-actin monoclonal antibody $(1: 1,000$; cat. no. ab6276; Abcam). Following incubation overnight at $4^{\circ} \mathrm{C}$ with primary antibodies, the membranes were washed with PBST three times for $10 \mathrm{~min}$ and were then incubated with mouse anti-rabbit monoclonal secondary antibody (1:10,000; cat. no. 5127; Cell Signaling Technology, Inc.) for $2 \mathrm{~h}$ at room temperature. Following development with an enhanced chemiluminescence (ECL) detection system, the western blot analysis results were analyzed using Image 1.41 software (National Institutes of Health).

Statistical analysis. All data are presented as the means \pm standard deviation and each experiment was repeated $a \geq 3$ times and data shown are representative experiments. The statistical analyses were performed using SPSS 21.0 software (IBM Corp., Armonk, NY, USA). Differences between three or more groups were analyzed by one-way analysis of variance, followed by the Student-Newman-Keuls post-hoc test for multiple comparisons. $\mathrm{P}<0.05$ was considered to indicate a statistically significant difference.

\section{Results}

Liraglutide improves spatial learning and memory in the DM group. In the present study, the MWM test was used to assess alterations in spatial learning and memory in rats. As shown in Fig. 1A, T2DM induced spatial learning impairment compared with in the control group, whereas administration of liraglutide caused a decrease in escape latency compared with in the DM group, particularly in the DM + Lira-H group. After 4 days, the platform was removed, and time spent in the target quadrant was reduced in the DM group compared with in the control group (Fig. 1B). Conversely, time in the quadrant in the
$\mathrm{DM}+$ Lira-L group was prolonged compared with in the DM group. Treatment with $200 \mu \mathrm{g} / \mathrm{kg}$ liraglutide exhibited the most significant improvement in the spatial probe test $(\mathrm{P}<0.05)$.

Treatment with liraglutide demonstrates significant protective effects against neuronal cell loss. Nissl staining was conducted to investigate neuronal alterations in the hippocampal CA1 region of rats in each group (Fig. 2). Hippocampal neurons in the DM group were characterized by marked shrinkage of neurocyte bodies, the presence of pyknotic pyramidal cells and the loss of nuclei (Fig. 2B). Conversely, in the control group, neurons were neatly arranged, and were conically shaped with a plump cytoplasm and round clear nucleus with prominent nucleoli (Fig. 2A). Treatment with liraglutide decreased DM-induced cell loss and pyknosis; however, certain degenerative cells with morphological alterations were still observed (Fig. 2C-E). These findings suggested that liraglutide treatment exerted protective effects against DM-induced neurotoxicity.

Liraglutide treatment promotes autophagy in the hippocampus of DM rats. The expression levels of autophagy-associated proteins, including Beclin-1, LC-3 II and LC-3 I, were detected in the hippocampus of DM rats in the present study. As shown in Fig. 3, a significant decrease occurred in the hippocampal levels of Beclin-1 and LC-3 II/LC-3 I in the DM group compared with in the control group $(\mathrm{P}<0.01)$. Furthermore, treatment with liraglutide significantly increased the expression levels of Beclin-1 and LC-3 II/LC-3 I compared with in the DM group, thus suggesting that autophagy may be involved in its effects $(\mathrm{P}<0.05)$. In addition, treatment with high dose of liraglutide mildly increased the protein expression levels of Beclin-1 and LC-3 II compared with in the Lira-L group $(\mathrm{P}<0.05)$.

Liraglutide improves activation of the PI3K/Akt pathway in $G K$ rats. The results of the present study indicated that the expression levels of PI3K and p-Akt were regulated by liraglutide. The protein expression levels of total Akt did not vary considerably among any of the groups. As shown in Fig. 4, western blotting demonstrated that the expression levels of PI3K and p-Akt were significantly decreased in hippocampal samples from the DM group compared with in the control (both $\mathrm{P}<0.01)$. However, the administration of liraglutide increased the expression levels of PI3K and p-Akt in a dose-dependent manner compared with in the DM group.

Liraglutide affects the expression levels of $p-A M P K$ and $p$-mTOR in the rat hippocampus. The expression levels of p-AMPK, AMPK, mTOR and p-mTOR in the hippocampus were measured by western blotting. Compared with in the control group, the expression levels of $\mathrm{p}$-AMPK were decreased in the DM group (Fig. 5A; $\mathrm{P}<0.01$ ), whereas they were upregulated by liraglutide treatment. Conversely, rats in the DM group exhibited significantly increased levels of p-mTOR compared with in the control group $(\mathrm{P}<0.01$; Fig. 5B). Treatment with liraglutide was also observed to attenuate the significantly increased expression of p-mTOR in the hippocampus of GK rats, particularly in the Lira-H group $(\mathrm{P}<0.05)$. These results indicated that administration 

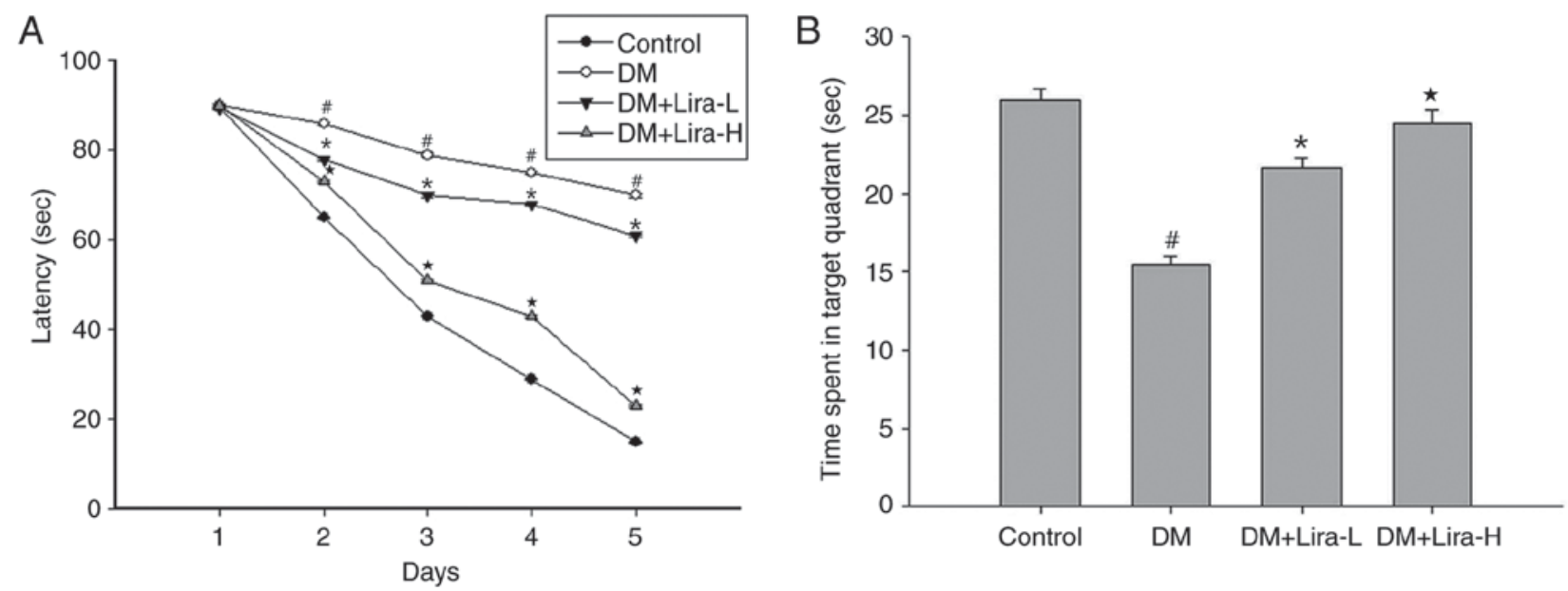

Figure 1. Effects of liraglutide on DM-induced deficits in spatial learning and memory were measured using the Morris water maze test. (A) Changes in daily escape latencies. (B) Time spent in the platform region in the probe trial. Values are expressed as the means \pm standard deviation. ${ }^{*} \mathrm{P}<0.05 \mathrm{vs}$. the control group. ${ }^{*} \mathrm{P}<0.05$ vs. the DM group. ${ }^{\star} \mathrm{P}<0.05$ vs. the Lira-L group. DM, diabetes mellitus; Lira-H, high-dose liraglutide; Lira-L, low-dose liraglutide.
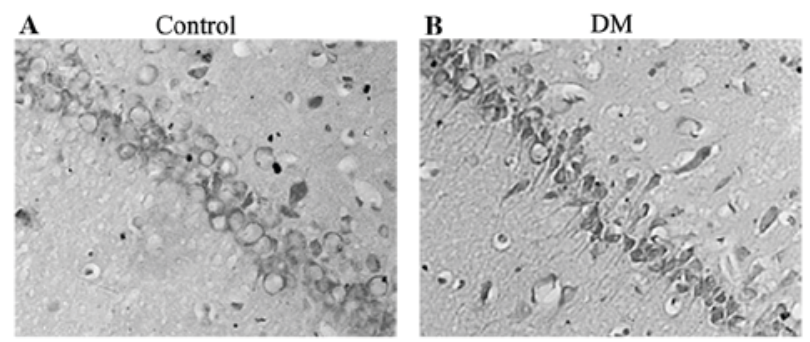

E
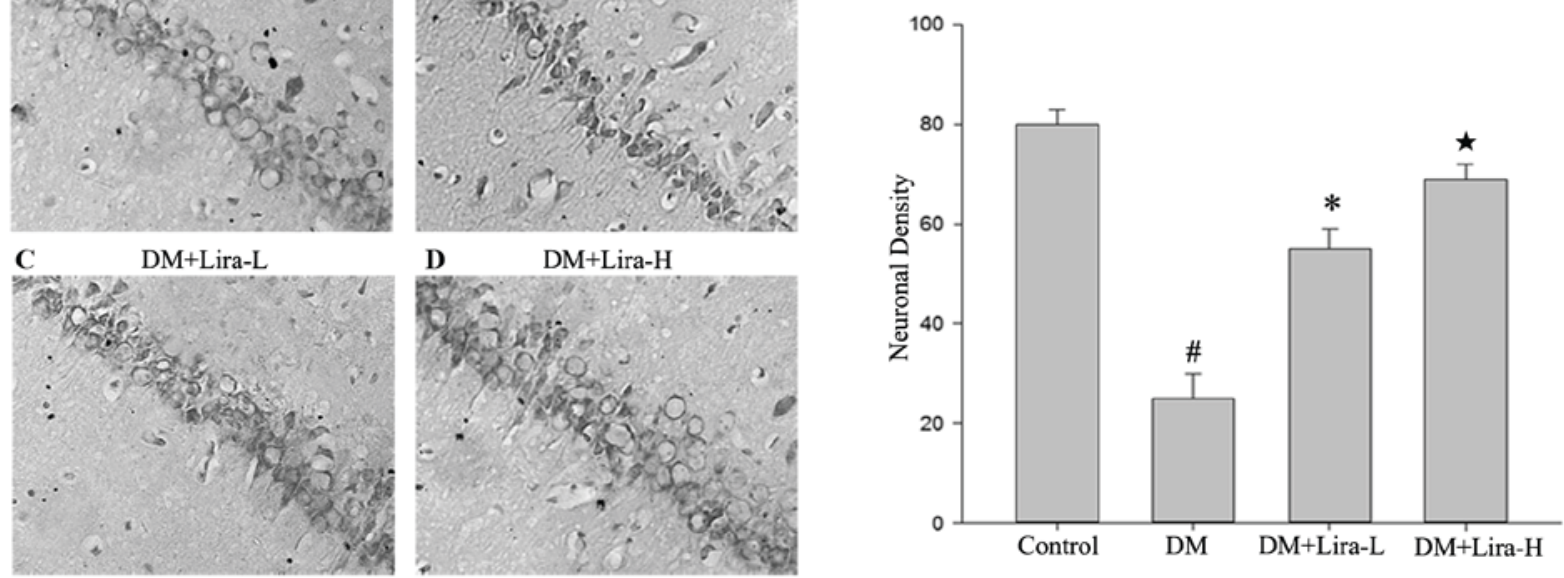

Figure 2. Histological analysis of the effects of liraglutide on neuronal injury in DM rats. Nissl staining was performed on sections from the hippocampal CA1 region. Magnification, x20. (A) Neurons containing round vesicular nuclei with significant nucleoli were observed in the control group, thus indicating the absence of degradation. (B) Alterations in the DM group were characterized by pronounced shrinkage of neuronal bodies, loss of nuclei and the presence of pyknotic pyramidal cells. DM-induced alterations were reduced in at the (C) DM + Lira-L and (D) DM + Lira-H groups. (E) Graphical representation of neuronal density in each group. ${ }^{~} \mathrm{P}<0.01$ vs. the control group. ${ }^{*} \mathrm{P}<0.05$ vs. the $\mathrm{DM}$ group. ${ }^{\star} \mathrm{P}<0.05$ vs. the Lira-L group. DM, diabetes mellitus; Lira-H, high-dose liraglutide; Lira-L, low-dose liraglutide.

of liraglutide may reverse the reduced expression of AMPK and overexpression of p-mTOR in T2DM in a dose-dependent manner.

Treatment with liraglutide attenuates the expression of apoptosis-associated proteins. The expression levels of caspase-3, Bax and Bcl-2 were detected in the hippocampus using western blot analysis. Compared with in the control group, the expression levels of caspase- 3 and Bax were significantly increased in the DM group (Fig. 6; $\mathrm{P}<0.01$ ), whereas they were downregulated by liraglutide administration. Conversely, the protein expression levels of $\mathrm{Bcl}-2$ were decreased in the DM group compared with the control group (Fig. 6). In liraglutide-treated (75 and $200 \mu \mathrm{g} / \mathrm{kg}$ ) rats, the expression levels of $\mathrm{Bcl}-2$ were increased compared with in the DM group. Therefore, Lira-L ( $75 \mu \mathrm{g} / \mathrm{kg}$ ) may markedly inhibit neuronal apoptosis. In addition, Lira-H $(200 \mu \mathrm{g} / \mathrm{kg})$ did not exhibit a more pronounced effect on the inhibition of apoptosis compared with Lira-L.

\section{Discussion}

DACD is a neurodegenerative disease that demonstrates a gradual progression of pathogenicity. The clinical manifestations of DACD include memory and learning impairment, disorientation, visual impairment, impairment of planning and executive functions, and personality and behavioral alterations (25). DACD is a common but easily neglected CNS complication of DM. Although numerous animal studies and clinical trials have been conducted regarding DACD $(26,27)$, 
A
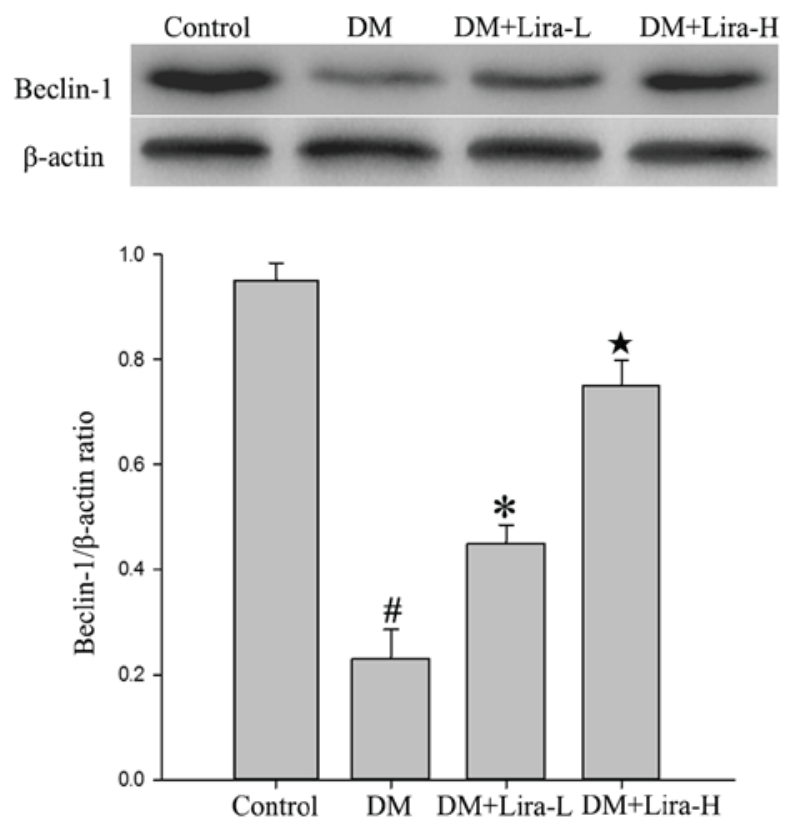

B
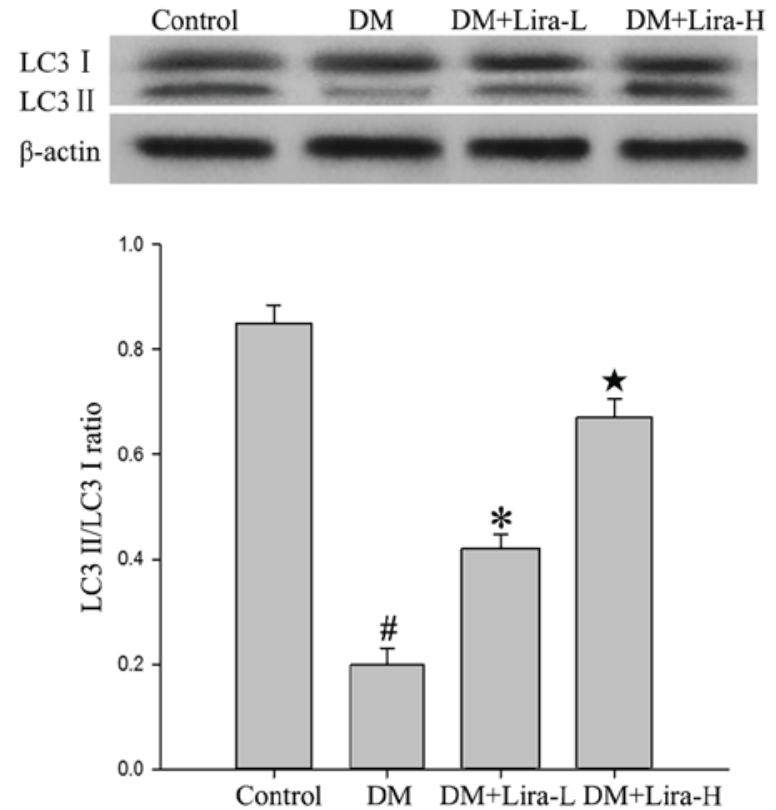

Figure 3. Effects of liraglutide on the expression levels of autophagy-associated proteins, as determined by western blotting of hippocampal tissues. $\beta$-actin was used as an internal control. Expression of (A) Beclin-1 and (B) LC-3 II was determined by western blotting. " P $<0.01$ vs. the control group. "P $<0.05$ vs. the DM group. ${ }^{\star} \mathrm{P}<0.05$ vs. the Lira-L group. DM, diabetes mellitus; LC-3, microtubule-associated protein light chain 3; Lira-H, high-dose liraglutide; Lira-L, low-dose liraglutide.

A
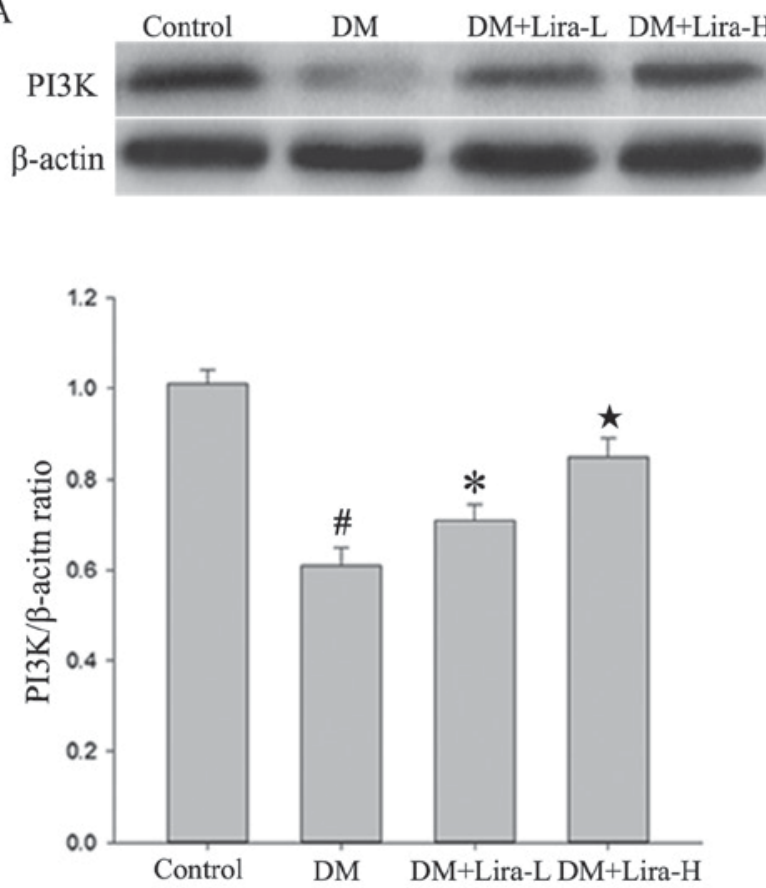

$\mathrm{B}$
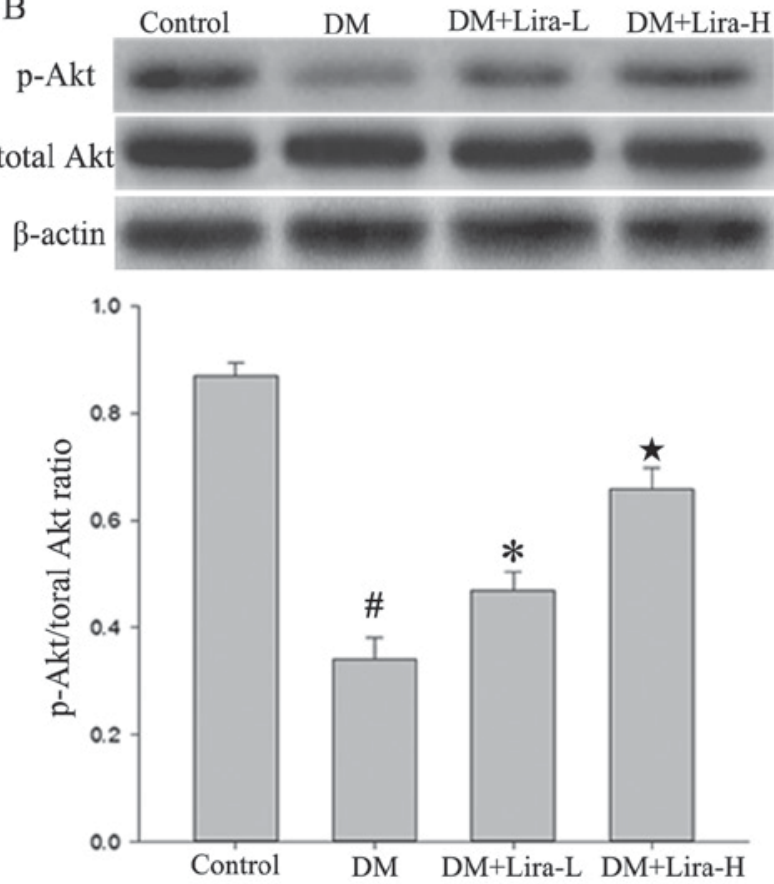

Figure 4. Effects of liraglutide on the PI3K/Akt pathway, as determined by western blotting of hippocampal tissues. $\beta$-actin was used as an internal control. Expression of (A) PI3K, and (B) total Akt and p-Akt was determined by western blotting. ${ }^{~} \mathrm{P}<0.01$ vs. the control group. ${ }^{*} \mathrm{P}<0.05$ vs. the $\mathrm{DM}$ group. ${ }^{\star} \mathrm{P}<0.05$ vs. the Lira-L group. Akt, protein kinase B; DM, diabetes mellitus; Lira-H, high-dose liraglutide; Lira-L, low-dose liraglutide; p-Akt, phosphorylated-Akt; PI3K, phosphoinositide 3-kinase.

the underlying pathogenesis remains unclear, and associated interventions or treatments have yet to be identified.

In recent years, studies have reported that autophagy serves an important role in the pathogenesis of DM-induced cognitive deficits and other complications $(28,29)$. Autophagy is a highly conserved metabolic process that is present in eukaryotic cells. Autophagy leads to the degradation of cytoplasmic components in lysosomes and has recently attracted considerable attention due to its association with DM-induced cognitive deficits (30). Observed autophagy impairments in the hippocampus of GK rats may 
A
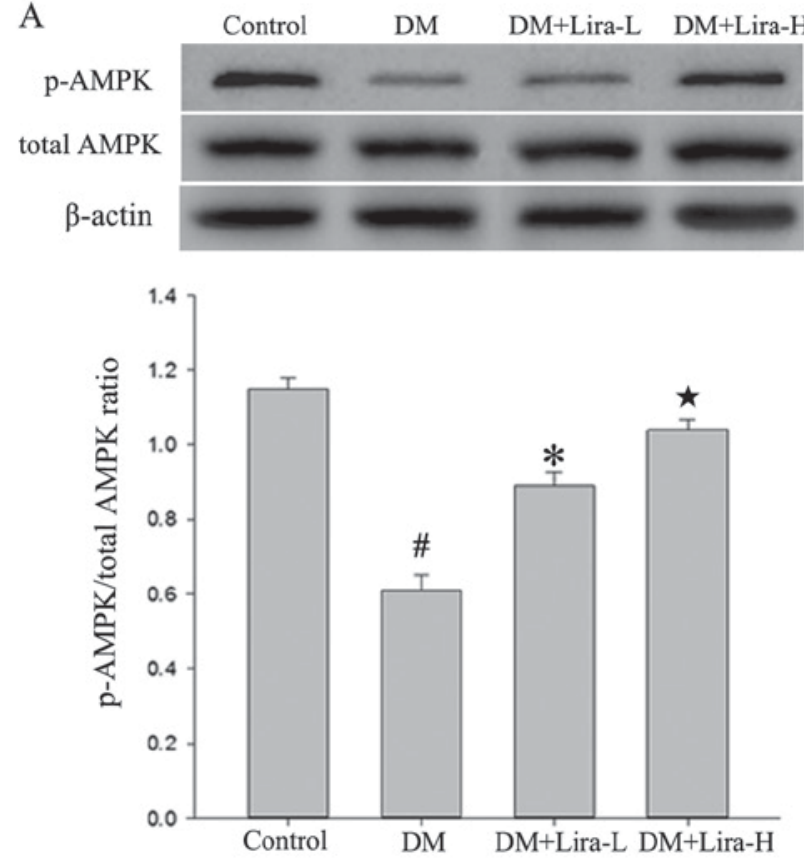

B p-mTOR total mTOR $\beta$-actin
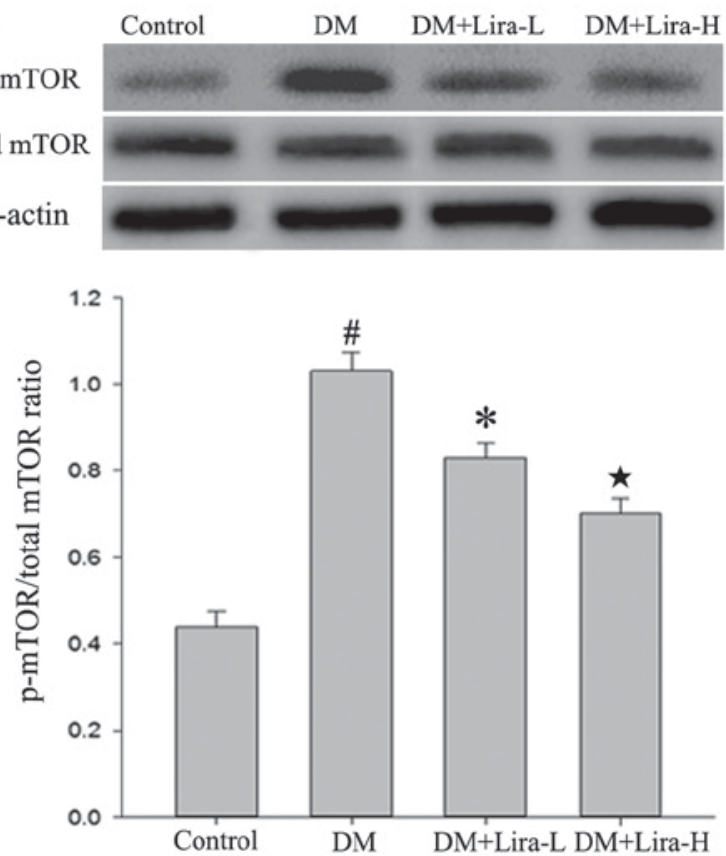

Figure 5. Effects of liraglutide on AMPK and mTOR expression, as determined by western blotting of hippocampal tissues. $\beta$-actin was used as an internal control. Expression of (A) AMPK and p-AMPK, and (B) mTOR and p-mTOR was determined by western blotting. "P $<0.01$ vs. the control group. "P<0.05 vs. the DM group. ${ }^{\star} \mathrm{P}<0.05$ vs. the Lira-L group. AMPK, AMP-activated protein kinase; DM, diabetes mellitus; Lira-H, high-dose liraglutide; Lira-L, low-dose liraglutide; mTOR, mammalian target of rapamycin; p-, phosphorylated.

A

\section{Control \\ $\mathrm{DM}$ \\ DM+Lira-L DM+Lira-H}

Caspase-3

$\beta$-actin

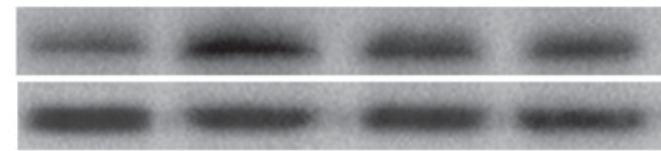

B

\section{Bax \\ Bcl-2 \\ $\beta$-actin}
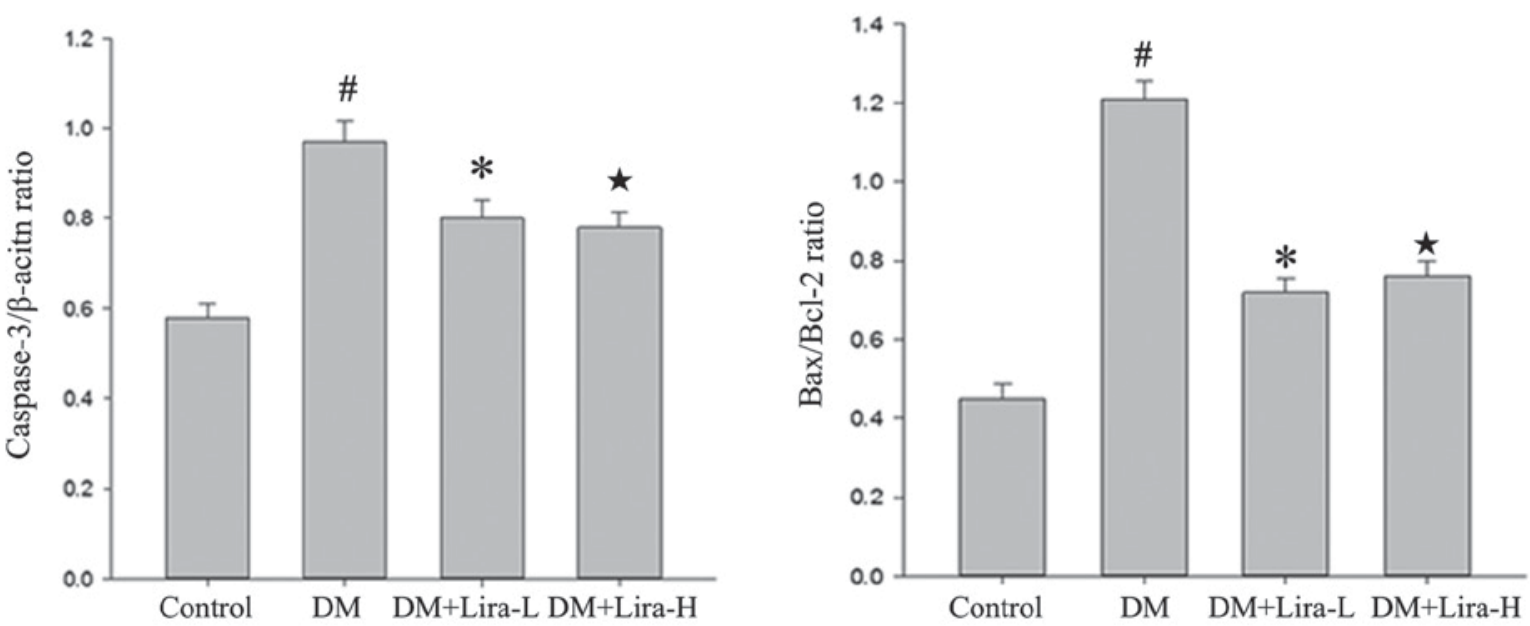

Figure 6. Effects of liraglutide on the expression levels of apoptosis-associated proteins, as determined by western blotting of hippocampal tissues. $\beta$-actin was used as an internal control. Expression of (A) caspase-3, and (B) Bax and Bcl-2 in western blotting. "P<0.01 vs. the control group. "P<0.05 vs. the DM group. ${ }^{\star} \mathrm{P}<0.05$ vs. the Lira-L group. Bax, Bcl-2-associated X protein; Bcl-2, B-cell lymphoma 2; DM, diabetes mellitus; Lira-H, high-dose liraglutide; Lira-L, low-dose liraglutide.

result in a reduction in hippocampal cell viability and dendritic spine density (31). Numerous hypotheses have been proposed to explain the molecular mechanisms by which autophagy is modulated in diabetic models. For example, the AMPK signaling pathway may exert a protective effect in high glucose-induced neuronal apoptosis via the induction of autophagy (32). Another 
study provided evidence to suggest that disturbance to endoplasmic reticulum stress may mediate downregulation of the Akt/tuberous sclerosis complex/mTOR pathway, thus resulting in the deactivation of mTOR, which causes downregulation of autophagy signaling (33). Hyperglycemia-associated oxidative stress can upregulate the reactive oxygen species-extracellular signal regulated kinase/c-Jun N-terminal kinase-p53 pathway, thus resulting in activation of autophagic signaling, which may induce mitochondrial loss in diabetic GK rats (34). The diversity of these examples could be explained by the fact that the observed autophagic alterations may be the net effect of numerous pathways.

GLP-1 receptor agonists are a novel class of hypoglycemic agents used as monotherapy or in combination with other antidiabetic compounds. GLP-1 receptor agonists directly activate the GLP-1 receptor on the $\beta$ cell membrane and promote its binding to GLP-1, which is released from L cells of the small intestine (35). It has previously been reported that GLP-1 receptor agonists have been used to improve cognitive functions in diabetic models. In a previous study, administration of exenatide reduced Tau hyperphosphorylation in the hippocampus of T2DM rats via insulin signaling and the PI3K/Akt/glycogen synthase kinase-3 (GSK-3) pathway (13). In addition, liraglutide, which is an efficient receptor agonist, can cross the blood-brain barrier, restrain hippocampal neuronal death and impede the reduced phosphorylation of Akt and p70S6K in the hippocampus of streptozotocin-treated animals (36). Liraglutide may also elicit a beneficial influence on synaptic plasticity in ob/ob mice with severe obesity, and improve hippocampal neurogenesis and neuronal survival partially by increasing the expression of achaete-scute family BHLH transcription factor 1 (37).

In the present study, liraglutide was chosen as the target therapeutic drug and diabetic GK rats were used to screen the effective doses. The results of the present study demonstrated that rats suffering from DM exhibited a decline in memory performance. Treatment for 28 days with $75 \mu \mathrm{g} / \mathrm{kg}$ liraglutide ameliorated cognitive deficits. In addition, the higher dose of liraglutide $(200 \mu \mathrm{g} / \mathrm{kg})$ demonstrated a protective effect on the DM model. These results displayed a dose-dependent effect of liraglutide on memory. In addition, the results demonstrated that liraglutide increased Beclin-1 and LC-3 II/LC-3 I expression compared with the DM group, which represented activation of autophagy. Research increasingly demonstrates that liraglutide can effectively mediate the process of autophagy in diverse tissues or cells affected by high glucose levels. In rat INS-1 $\beta$-cells, liraglutide has been revealed to enhance autophagy, and suppression of autophagy decreased the anti-apoptotic effects of liraglutide under high glucose conditions (22). Furthermore, a recent study demonstrated that liraglutide treatment protected cardiomyocytes from high glucose-induced apoptosis, which is accompanied by a notable increase in autophagy via activation of the Rap guanine nucleotide exchange factor 3/Akt signaling pathway (38). Furthermore, in accordance with potential liraglutide-induced autophagy in the hippocampus of T2DM rats, the present study detected a slightly higher level of PI3K/Akt and p-AMPK expression following liraglutide administration in GK rats. In addition, liraglutide reversed the increased levels of p-mTOR in the hippocampus of GK rats. These findings indicated that liraglutide administration following neuronal damage may inhibit expression of $\mathrm{p}$-mTOR via activation of the PI3K/Akt and AMPK signaling pathway, thus resulting in enhanced autophagic signaling and improved cognitive function, which is similar to the previously reported effects of Exendin-4 in a T2DM rat model (23).

There are numerous types of cell death, including necrotic, apoptotic and autophagic cell death. A complex interaction exists between autophagy and apoptosis, and both can be activated by numerous stress stimuli, regulatory molecules, and may even share common pathways that exert neuroprotective effects. Under certain circumstances, autophagy is a stress adaptation that inhibits apoptosis and prevents cell death; however, in other cellular settings it constitutes another pathway of cell death. The results of the present study demonstrated that a low dose of liraglutide may be sufficient to reduce the expression of caspase-3 and $\mathrm{Bax}$, and to increase the expression of $\mathrm{Bcl}-2$, thus resulting in the reduction of apoptosis, which is similar to the results of a previous study by Badawi et al (39). According to the results of the present study, administration of liraglutide may activate autophagy while suppressing apoptosis. Regulation of autophagy, PI3K/Akt/GSK3 $\beta$ activation and several other mechanisms are all possible mechanisms underlying the decreased expression of caspase-3 and Bax following liraglutide administration, which was confirmed by previous studies $(23,40,41)$. However, the probable mechanism underlying the interaction of autophagy and apoptosis in the present study was not determined. In future research, the crosstalk between autophagy and apoptosis, and its underlying molecular mechanism, should be investigated.

In conclusion, the present study demonstrated that administration of liraglutide decreased the expression of apoptosis-associated proteins. In addition, it activated the AMPK and PI3K/Akt signaling pathways in the hippocampus of GK rats, and promoted autophagy, ultimately providing protection against chronic T2DM-associated learning and memory impairments. The present study provided evidence that may facilitate the development of liraglutide as a promising preventative or therapeutic strategy for the treatment of DM-induced cognitive impairment.

\section{Acknowledgements}

The authors would like to thank all teachers in the Animal Experiment Center of North China University of Science and Technology (Tangshan, China) for their technical support for the experiments.

\section{Funding}

The present study was supported by a Project of Hebei Provincial Natural Science fund (grant no. H2015105083).

\section{Availability of data and materials}

All data generated or analyzed during this study are included in this published article.

\section{Authors' contributions}

YY, HF and GX conceived and designed the study. YZhe and YZha performed the experiments. YY wrote the manuscript. 
JT, DZ, GZ and JX analyzed the data. All authors read and approved the final manuscript.

\section{Ethics approval and consent to participate}

The animal experiments were conducted in compliance with the guidelines for the Care and Use of Laboratory Animals (National Institutes of Health, Bethesda, MD, USA). The present study was approved by the experimental ethics committee of North China University of Science and Technology (Tangshan, China).

\section{Patient consent for publication}

Not applicable.

\section{Competing interests}

The authors declare that they have no competing interests.

\section{References}

1. Dobretsov M, Romanovsky D and Stimers JR: Early diabetic neuropathy: Triggers and mechanisms. World J Gastroenterol 13: 175-191, 2007.

2. McCrimmon RJ, Ryan CM and Frier BM: Diabetes and cognitive dysfunction. Lancet 379: 2291-2299, 2012.

3. Mijnhout GS, Scheltens P, Diamant M, Biessels GJ, Wessels AM, Simsek S, Snoek FJ and Heine RJ: Diabetic encephalopathy: A concept in need of a definition. Diabetologia 49: 1447-1448, 2006.

4. Carvalho C, Santos MS, Oliveira CR and Moreira PI: Alzheimer's disease and type 2 diabetes-related alterations in brain mitochondria, autophagy and synaptic markers. Biochim Biophys Acta 1852: 1665-1675, 2015.

5. Feinkohl I, Price JF, Strachan MW and Frier BM: The impact of diabetes on cognitive decline: Potential vascular, metabolic and psychosocial risk factors. Alzheimers Res Ther 7: 46, 2015.

6. Vagelatos NT and Eslick GD: Type 2 diabetes as a risk factor for Alzheimer's disease: The confounders, interactions and neuropathology associated with this relationship. Epidemiol Rev 35: 152-160, 2013.

7. Muriach M, Flores-Bellver M, Romero FJ and Barcia JM: Diabetes and the brain: Oxidative stress, inflammation and autophagy. Oxid Med Cell Longev 2014: 102158, 2014.

8. Guan ZF, Tao YH, Zhang XM, Guo QL, Liu YC, Zhang Y, Wang YM, Ji G, Wu GF, Wang NN, et al: G-CSF and cognitive dysfunction in elderly diabetic mice with cerebral small vessel disease: Preventive intervention effects and underlying mechanisms. CNS Neurosci Ther 23: 462-474, 2017.

9. Kong FJ, Ma LL, Guo JJ, Xu LH, Li Y and Qu S: Endoplasmic reticulum stress/autophagy pathway is involved in diabetes-induced neuronal apoptosis and cognitive decline in mice. Clin Sci 132: $111-125,2018$

10. Qian M, Fang X and Wang X: Autophagy and inflammation. Clin Transl Med 6: 24, 2017.

11. Guan ZF, Zhou XL, Zhang XM, Zhang Y, Wang YM, Guo QL Ji G, Wu GF, Wang NN, Yang H, et al: Beclin-1-mediated autophagy may be involved in the elderly cognitive and affective disorders in streptozotocin-induced diabetic mice. Transl Neurodegener 5: 22, 2016.

12. Palleria C, Leporini C, Maida F, Succurro E, De Sarro G, Arturi F and Russo E: Potential effects of current drug therapies on cognitive impairment in patients with type 2 diabetes. Front Neuroendocrinol 42: 76-92, 2016.

13. Goto H, Nomiyama T, Mita T, Yasunari E, Azuma K, Komiya K, Arakawa M, Jin WL, Kanazawa A, Kawamori R, et al: Exendin-4, a glucagon-like peptide-1 receptor agonist, reduces intimal thickening after vascular injury. Biochem Biophys Res Commun 405: 79-84, 2011.

14. Chen S, Liu AR, An FM, Yao WB and Gao XD: Amelioration of neurodegenerative changes in cellular and rat models of diabetes-related Alzheimer's disease by exendin-4. Age 34: $1211-1224,2012$.
15. Solmaz V, Çınar BP, Yiğittürk G, Çavuşoğlu T, Taşkıran D and Erbaş O: Exenatide reduces TNF- $\alpha$ expression and improves hippocampal neuron numbers and memory in streptozotocin treated rats. Eur J Pharmacol 765: 482-487, 2015.

16. Zheng T, Qin L, Chen B, Hu X, Zhang X, Liu Y, Liu H, Qin S, Li G and Li Q: Association of plasma DPP4 activity with mild cognitive impairment in elderly patients with type 2 diabetes: Results from the GDMD study in China. Diabetes Care 39: 1594-1601, 2016.

17. Chen B, Zheng T, Qin L, Hu X, Zhang X, Liu Y, Liu H, Qin S, Li G and Li Q: Strong association between plasma dipeptidyl peptidase-4 activity and impaired cognitive function in elderly population with normal glucose tolerance. Fron Aging Neurosci 9: 247, 2017.

18. Zheng T, Liu H, Qin L, Chen B, Zhang X, Hu X, Xiao L and Qin S: Oxidative stress-mediated influence of plasma DPP4 activity to BDNF ratio on mild cognitive impairment in elderly type 2 diabetic patients: Results from the GDMD study in China. Metabolism: March 21, 2018 (Epub ahead of print).

19. Jacobsen LV, Hindsberger $C$, Robson $R$ and Zdravkovic M: Effect of renal impairment on the pharmacokinetics of the GLP-1 analogue liraglutide. Br J Clin Pharmacol 68: 898-905, 2009.

20. McClean PL and Hölscher C: Liraglutide can reverse memory impairment, synaptic loss and reduce plaque load in aged APP/PS1 mice, a model of Alzheimer's disease. Neuropharmacology 76: 57-67, 2014.

21. Hansen HH, Fabricius K, Barkholt P, Niehoff ML, Morley JE, Jelsing J, Pyke C, Knudsen LB, Farr SA and Vrang N: The GLP-1 receptor agonist liraglutide improves memory function and increases hippocampal CA1 neuronal numbers in a senescence-accelerated mouse model of Alzheimer's disease. J Alzheimers Dis 46: 877-888, 2015.

22. Miao X, Gu Z, Liu Y, Jin M, Lu Y, Gong Y, Li L and Li C: The glucagon-like peptide-1 analogue liraglutide promotes autophagy through the modulation of 5'-AMP-activated protein kinase in INS-1 $\beta$-cells under high glucose conditions. Peptides 100: 127-139, 2018.

23. Candeias E, Sebastião I, Cardoso S, Carvalho C, Santos MS, Oliveira CR, Moreira PI and Duarte AI: Brain GLP-1/IGF-1 signaling and autophagy mediate exendin-4 protection against apoptosis in type 2 diabetic rats. Mol Neurobiol 55: 4030-4050, 2018.

24. National Research Council (US), Institute for Laboratory Animal Research: Guide for the care and use of laboratory animals. The National Academies Press, Washington, DC, 1996.

25. Ryan CM, van Duinkerken E and Rosano C: Neurocognitive consequences of diabetes. Am Psychol 71: 563-576, 2016.

26. Stranahan AM: Models and mechanisms for hippocampal dysfunction in obesity and diabetes. Neuroscience 309: 125-139, 2015.

27. Yates KF, Sweat V, Yau PL, Turchiano MM and Convit A: Impact of metabolic syndrome on cognition and brain: A selected review of the literature. Arterioscler Thromb Vasc Biol 32: 2060-2067, 2012.

28. de Faria JM, Duarte DA, Montemurro C, Papadimitriou A, Consonni SR and Lopes de Faria JB: Defective autophagy in diabetic retinopathy defective autophagy. Invest Ophthalmol Vis Sci 57: 4356-4366, 2016.

29. Ma LY, Lv YL, Huo K, Liu J, Shang SH, Fei YL, Li YB, Zhao BY, Wei M, Deng YN and Qu QM: Autophagy-lysosome dysfunction is involved in A $\beta$ deposition in STZ-induced diabetic rats. Behav Brain Res 320: 484-493, 2017.

30. Guan ZF, Zhou XL, Zhang XM, Zhang Y, Wang YM, Guo QL, $\mathrm{Ji} \mathrm{G}$, Wu GF, Wang NN, Yang $\mathrm{H}$, et al: Beclin-1-mediated autophagy may be involved in the elderly cognitive and affective disorders in streptozotocin-induced diabetic mice. Transl Neurodegener 5: 22, 2016.

31. Li XH, Xin X, Wang Y, Wu JZ, Jin ZD, Ma LN, Nie CJ, Xiao X, $\mathrm{Hu} \mathrm{Y}$ and Jin MW: Pentamethylquercetin protects against diabetes-related cognitive deficits in diabetic Goto-Kakizaki rats. J Alzheimers Dis 34: 755-767, 2013.

32. Xue H, Ji Y, Wei S, Yu Y, Yan X, Liu S, Zhang M, Yao F, Lan X and Chen L: HGSD attenuates neuronal apoptosis through enhancing neuronal autophagy in the brain of diabetic mice: The role of AMP-activated protein kinase. Life Sci 153: 23-34, 2016.

33. Qin L, Wang Z, Tao L and Wang Y: ER stress negatively regulates AKT/TSC/mTOR pathway to enhance autophagy. Autophagy 6: 239-247, 2010. 
34. Yan J, Feng Z, Liu J, Shen W, Wang Y, Wertz K, Weber P, Long $\mathbf{J}$ and Liu J: Enhanced autophagy plays a cardinal role in mitochondrial dysfunction in type 2 diabetic Goto-Kakizaki (GK) rats: Ameliorating effects of (-)-epigallocatechin-3-gallate. J Nutr Biochem 23: 716-724, 2012.

35. Holz GG IV, Kiihtreiber WM and Habener JF: Pancreatic beta-cells are rendered glucose-competent by the insulinotropic hormone glucagon-like peptide-1(7-37). Nature 36: 362-365, 1993

36. Palleria C, Leo A, Andreozzi F, Citaro R, Lannone M, Spiga R, Sesti G, Constanti A, De Sarri G, Arturi F and Russo E: Liraglutide prevents cognitive decline in a rat model of streptozotocin-induced diabetes independently from its peripheral metabolic effects. Behav Brain Res 321: 157-169, 2017.

37. Porter WD, Flatt PR, Hölscher C and Gault VA: Liraglutide improves hippocampal synaptic plasticity associated with increased expression of Mash1 in ob/ob mice. Int J Obes (Lond) 37: 678-684, 2013.
38. Wu XM, Ou QY, Zhao W, Liu J and Zhang H: The GLP-1 analogue liraglutide protects cardiomyocytes from high glucose-induced apoptosis by activating the Epac-1/Akt pathway. Exp Clin Endocrinol Diabetes 122: 608-614, 2014.

39. Badawi GA, Abd El Fattah MA, Zaki HF and EI Sayed MI: Sitagliptin and liraglutide reversed nigrostriatal degeneration of rodent brain in rotenone-induced Parkinson's disease. Inflammopharmacology 25: 369-382, 2017.

40. Abbas NAT and Kabil SL: Liraglutide ameliorates cardiotoxicity induced by doxorubicin in rats through the Akt/GSK-3 $\beta$ signaling pathway (J). Archiv Für Exp Pathol Und Pharmakol: $1-9,2017$.

41. Zhu H, Zhang Y, Shi Z, Lu D, Li T, Ding Y, Ruan Y and Xu A: The neuroprotection of liraglutide against ischaemia-induced apoptosis through the activation of the PI3K/AKT and MAPK Pathways. Sci Rep 6: 26859, 2016. 